\title{
Mass Transfer Measurements behind an Orifice in a Circular Pipe Flow for Various Combinations of Swirl Intensity and Orifice Bias*
}

\author{
Tsuyoshi TAKANO**, Takayuki YAMAGATA***, Akihiro ITO** \\ and Nobuyuki FUJISAWA*** \\ ${ }^{* *}$ Graduate School of Science and Technology, Niigata University \\ 8050 Ikarashi 2-Nocho, Nishi-ku, Niigata, 950-2181, Japan \\ ***Visualization Research Center, Niigata University \\ 8050 Ikarashi 2-Nocho, Nishi-ku, Niigata, 950-2181, Japan \\ E-mail: fujisawa@eng.niigata-u.ac.jp
}

\begin{abstract}
A pipe-wall thinning phenomenon behind an orifice in a circular pipe is studied by measuring the mass transfer using the naphthalene sublimation method. An attention is placed on an asymmetric increase of local mass transfer behind the orifice, which is combined with the influence of swirling flow and the orifice bias. The present results indicate that the mass transfer is generally increased with an increase in swirl intensity. However, it becomes asymmetric by the combined effect of the orifice bias for the bias larger than $0.4 \%$ of the pipe diameter and the swirl intensity larger than approximately 0.2 . The enhanced mass transfer is found on the shorter orifice side behind a biased orifice, and the maximum mass transfer is increased $40 \%$ in comparison with the case without swirl and orifice bias.
\end{abstract}

Key words: Flow Accelerated Corrosion, Pipe-Wall Thinning, Mass Transfer, Orifice, Swirling Flow, Nuclear Power Plant

\section{Introduction}

Pipe wall thinning in a pipeline of nuclear/fossil power plants is an important topic of interests from the point of safety management and maintenance of highly aged power plants. It is well known that one of the causes of the pipe-wall thinning is due to the flow accelerated corrosion (FAC), which is the corrosion phenomenon of the pipe-wall material accelerated by the flow turbulence. This phenomenon often occurs in highly turbulent flow region in the pipeline, such as in the pipe behind orifice, $\mathrm{T}$ junction, elbow, and so on. Although the fundamental aspects of this phenomenon has been studied experimentally and numerically, this phenomenon has not been fully understood yet due to the complexity of this phenomenon related to the water chemistry, fluid mechanics, heat and mass transfer and solid mechanics of the pipe-wall material, and so on.

In 2004, a pipe-wall thinning accident due to FAC occurred in the pipeline of Mihama nuclear power plant in Japan. According to the report by NISA [1], the pipe-wall thinning appeared on the pipe wall behind the orifice, which was found over the pipe wall for several pipe diameters downstream from the orifice. An interesting point was that the pipe-wall thinning became asymmetric about the pipe axis, in spite of basically symmetrical arrangement of the circular orifice in a circular pipe. It should be noted that the rupture accident of the prototype pipeline was expected to be accelerated by the asymmetric 
pipe-wall thinning, because the pipe-wall thinning on one side was much larger than the other side of the pipe.

Although there are many experimental and numerical studies on the flow behind the orifice [2-10], the occurrence of asymmetrical flow behind the orifice has not been studied in literature except for a few recent studies on the flow behind the biased orifice [11-14]. Ohkubo et al. $[12,13]$ carried out the PIV measurement for the orifice flow and showed that the combined effect of the swirling flow and the small orifice bias of $0.7 \%$ can produce the asymmetric flow pattern behind the orifice. It should be mentioned that the orifice bias corresponds to the pipe diameter error, which is known to be $0.8 \%$ of the pipe diameter (JIS G3456). Therefore, the flow through an orifice is essentially unstable to the small disturbances caused by the minor asymmetry of the orifice. In this asymmetric flow, Takano et al. [14] showed that asymmetric mass transfer occurs behind the orifice by using the naphthalene sublimation method. From the point of view of pipe-wall thinning management, it is important to clarify the critical condition for the occurrence of the asymmetric mass transfer, which is closely related to the pipe-wall thinning.

The purpose of this study is to understand the critical condition for the occurrence of asymmetric flow pattern and mass transfer in the flow behind the orifice due to the combined effect of the swirling flow and the orifice bias, using the mass transfer measurements by the naphthalene sublimation method.

\section{Experimental methods}

\subsection{Experimental apparatus}

Figure 1 shows a schematic illustration of the experimental apparatus for mass transfer measurements in the flow behind an orifice. The experimental apparatus consists of a blower, a straight pipe section, a swirler and a test pipe with an orifice. The flow rate of the air was measured in the downstream of the test section using an orifice flow meter. Note that the flow rate was adjusted by the inverter by controlling the rotational speed of the blower. The Reynolds number of the flow through the pipe was set to $\operatorname{Re}(=U D / v)=1.9 \times$ $10^{4}$, where $U$ is the bulk velocity, $D(=56 \mathrm{~mm})$ is the pipe diameter, and $v$ is the kinematic

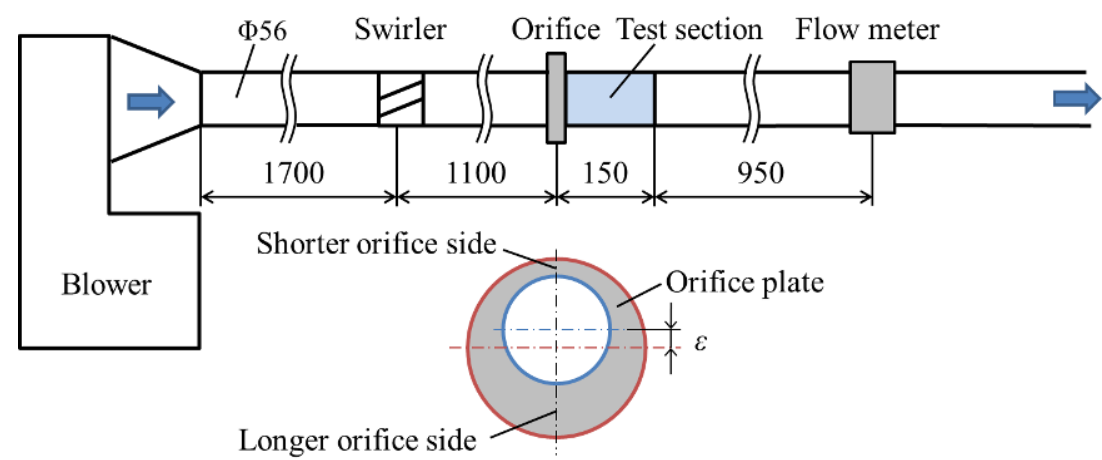

Fig. 1 Experimental apparatus for mass transfer measurement (unit in $\mathrm{mm}$ )
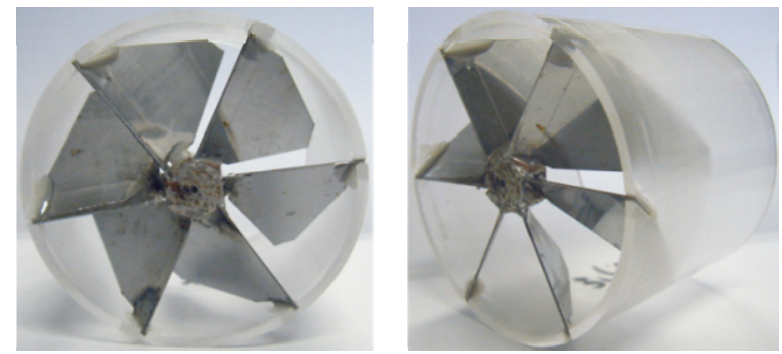

Fig. 2 Picture of swirler 
viscosity of the air. The swirling flow is generated by the swirler, which consists of six flat plates inclining at a certain angle to the circumferential direction. In the present experiment, three swirlers with plate angles $\alpha=15^{\circ}, 22.5^{\circ}$ and $30^{\circ}$ are made to study the influence of the swirl intensity on the mass transfer measurements. The picture of the swirler with plate angle $30^{\circ}$ is shown in Fig. 2.

The test section is made of aluminum material to keep the high circularity and smoothness by machining the surface. A circular orifice plate is attached to the flange structure in the test section. The orifice has a diameter ratio of 0.6 to meet the experimental condition of the prototype pipeline [1]. In order to obtain a certain orifice bias in the vertical direction, the position of the orifice plate was controlled by a screw in the flange structure. The magnitude of the orifice bias is set to $\varepsilon=0.4 \%, 0.8 \%$ and $1.2 \%$ of the pipe diameter $D$ in the present experiment. Note that the orifice bias $0.8 \%$ corresponds to the allowable pipe diameter error of the steel pipe for nuclear/fossil power plants (JIS G3456).

\subsection{Mass transfer measurement}

The measurement of mass transfer behind the orifice in a circular pipe was carried out by using the naphthalene sublimation method [15]. This method allows the evaluation of mass transfer coefficient $h$ on the naphthalene surface in the air flow. The mass transfer coefficient $h$ can be evaluated from the following equation;

$$
h=\frac{\rho_{s}}{\rho_{v}} \cdot \frac{\delta z}{\delta t}
$$

where $\delta z / \delta t$ is the thinning rate of the solid naphthalene in a unit time, $\rho_{s}$ is the density of solid naphthalene, $\rho_{v}$ is the density of vapour naphthalene. In the present study, the thinning rate $\delta z / \delta t$ was evaluated from the depth measurement by the confocal laser displacement sensor, and the densities of the solid and vapour naphthalene were evaluated from the temperature measurement in the air flow by a thermocouple, using the empirical formula of Ambrose et al. [16]. It was assumed that the surface temperature of naphthalene was approximated by the air flow temperature. The air flow temperature was controlled not lower than $293 \mathrm{~K}$. By evaluating the diffusion coefficient $D_{i}$ of the vapour naphthalene from the empirical formula of Goldstein-Chou [15], the Sherwood number $S h\left(=h D / D_{i}\right)$ is obtained.

Figure 3 shows the schematic illustration of the test section behind the orifice for measuring the thinning rate on the solid naphthalene. The test section consists of two separate semi-circular blocks, which are joined behind the orifice to form a circular pipe. The mass transfer measurements were carried out on the middle section along the centerline, where the naphthalene was casted in the circular grooves of the semi-circular blocks. The height of the groove is $2 \mathrm{~mm}$ and the length is $140 \mathrm{~mm}$ behind the orifice. The width of the circular groove is $84 \mathrm{~mm}$ in the circumferential direction, which is wide enough not to be influenced by the side wall of the groove. The molten naphthalene was casted into the groove on the semi-circular blocks. The melting temperature of the naphthalene is $353 \mathrm{~K}$. In order to cast the naphthalene into the groove, the aluminum test section was heated up to $360 \mathrm{~K}$ before casting the naphthalene. Then, both the test section and the casted naphthalene were gradually cooled down to the surrounding temperature. Finally, the surface of the naphthalene was polished by the emery papers to remove the surface roughness. The picture of the test section is shown in Fig. 4.

The mass transfer measurements were carried out along the centerline of each semi-circular block, using a confocal laser displacement sensor. This sensor had an accuracy of measurement less than $\pm 0.1 \mu \mathrm{m}$. The line traverse of the laser sensor was made by the 


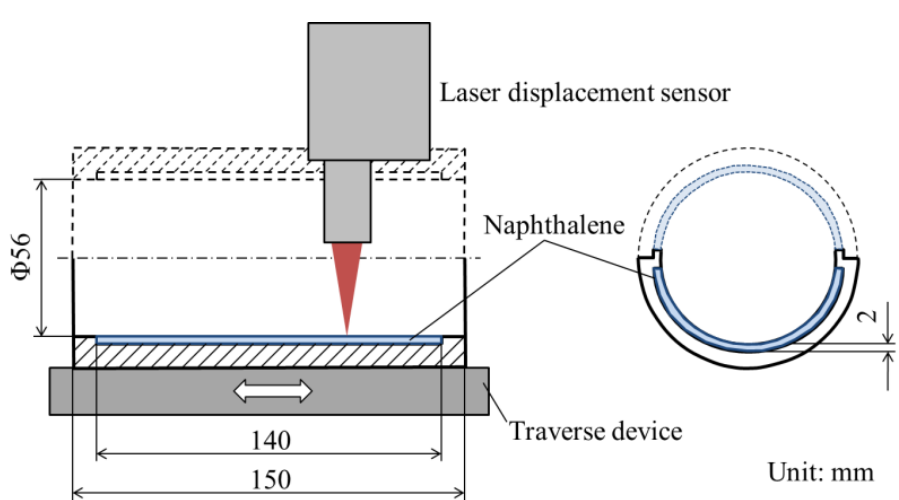

Fig. 3 Details of test section

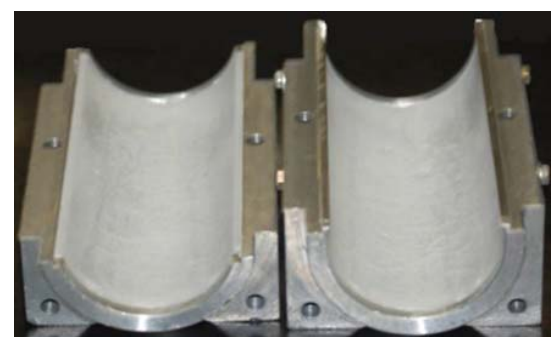

Fig. 4 Picture of test section

traversing equipment driven by a stepping motor, which allowed the measurement of thinning rate along the centerline on the top and bottom of the test section, where the maximum displacement of the orifice bias occurred. The thinning rate of the naphthalene was evaluated from the depth measurement of the naphthalene surface before and after the experiment. The duration time of the experiment was set to 30 minutes, which resulted in the average surface-height variation of 50 to $60 \mu \mathrm{m}$.

\section{Results and discussion}

\subsection{Swirl intensity}

The measurements of swirl intensity were carried out in the circular pipe without orifice. The experiments were conducted in the water tunnel using the standard PIV system [12]. For details of the experimental technique see Ref. [12]. Based on the mean velocity distribution measured in the two cross-sections of the pipe flow, the swirl intensity in the test section is evaluated [12]. Note that the swirl intensity is defined as follows [17]:

$$
S=\int_{0}^{R} u v_{\theta} r^{2} d r / R \int_{0}^{R} u^{2} r d r
$$

where $R$ : radius of the pipe, $r$ : distance from the pipe axis, $u$ : streamwise mean velocity, $v_{\theta}$ : circumferential mean velocity. The results are summarized in Table 1, which indicates that the swirl intensity ranges from $S=0$ to 0.3 in the present experiment. This range of the swirl intensity is in close agreement with that in the scaled-model-experiment of the prototype pipeline [1].

\subsection{Validation of mass transfer measurements}

In order to validate the mass transfer measurements by the naphthalene sublimation method, the mass transfer measurements were carried out in a circular pipe without orifice. 
Table 1 Relationship between plate angles $\alpha$ and swirl intensities $S$

\begin{tabular}{|c|c|c|c|c|}
\hline$\alpha$ & $0^{\circ}$ & $15^{\circ}$ & $22.5^{\circ}$ & $30^{\circ}$ \\
\hline$S$ & 0 & 0.09 & 0.16 & 0.25 \\
\hline
\end{tabular}

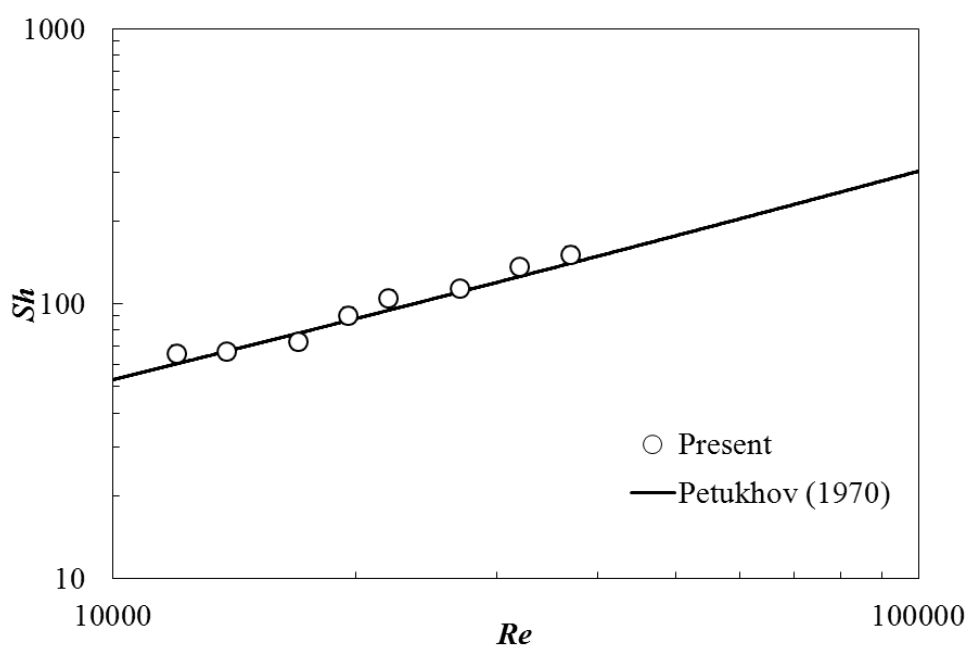

Fig. 5 Sherwood number $S h$ versus Reynolds number Re in circular pipe

Figure 5 shows the variations of the Sherwood number $S h$ with the Reynolds number $R e$, which is compared with the empirical formula of mass transfer in a circular pipe flow by Petukhov [18]. The present measurements are in close agreement with the empirical formula in the range of uncertainty of measurement, which is represented by the marker size in Fig. 5. It should be mentioned that the uncertainty of the mass transfer measurement is estimated as $7.3 \%$ with the confidence interval of $95 \%$.

\subsection{Mass transfer measurements in swirling flow}

Figure 6 shows the Sherwood number distributions behind the orifice without bias for various swirl intensities ranging from 0 to 0.3 , which are plotted against the distance $x / D$ from the orifice. The Sherwood number distribution without swirling flow shows a peak in the downstream distance $x / D=1-2$, and the maximum value reaches to $S h_{\max }=380$, which

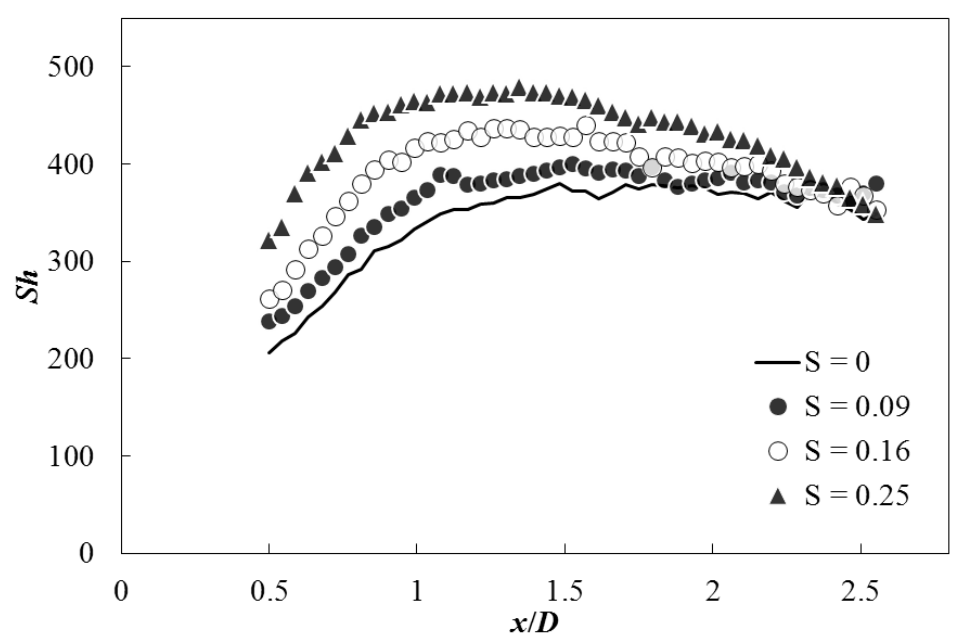

Fig. 6 Sherwood number distributions behind orifice without bias in swirling flow $(\operatorname{Re}=1.9$ $\times 10^{4}$ ) 
is about 4 times larger than that without orifice at the same Reynolds number $R e=1.9 \times$ $10^{4}$. Thus, the present result indicates that the mass transfer is greatly increased behind the orifice in comparison with that of the circular pipe flow without orifice. Note that the mass transfer ratio 4 agrees with the previous measurements of pipe-wall thinning behind the orifice [2], which suggests the effectiveness of the mass transfer measurements instead of the direct measurement of pipe-wall thinning of steel pipe. On the other hand, the Sherwood number distribution increases gradually with an increase in swirl intensity, and the position of the maximum Sherwood number moves upstream. Therefore, the swirling flow is considered influential on the Sherwood number distribution behind the orifice. Note that the Sherwood number distribution behind the orifice without bias is keeping symmetry with respect to the flow axis.

\subsection{Mass transfer measurements in swirling flow with orifice bias}

Figures 7(a)-(d) show the Sherwood number distributions behind the biased orifices for various swirl intensities $S=0$ (a), 0.09 (b), 0.16 (c), and 0.25 (d), respectively. Each figure shows the results for various biases of $\varepsilon=0.4 \%, 0.8 \%$, and $1.2 \%$ with respect to the pipe diameter. The Sherwood number distributions behind the biased orifices without swirl $(S=$ 0 ) show that the mass transfer does not change by the influence of the orifice bias in the range of the present study. This is true for the mass transfer behind the biased orifice with small swirl intensities of $S=0.09$ (b) and $S=0.16$ (c). On the other hand, the Sherwood number distributions with large swirl intensity of $S=0.25$ (d) indicate the variation of the mass transfer rate with respect to the orifice biases. The Sherwood number increases on the shorter orifice side with increasing the orifice bias and it decreases on the longer orifice side. It should be noted that the influence of the orifice bias is found even for the small bias as small as $0.4 \%$ of the pipe diameter. It seems that the position of the maximum Sherwood number moves slightly upstream by increasing the orifice bias on the shorter orifice side and vice versa on the longer orifice side. These results indicate that the asymmetric distribution of mass transfer is generated behind the biased orifice, when the swirl intensity increases beyond approximately $S=0.2$ and the orifice bias is larger than $0.4 \%$ of the pipe diameter. This result is supported by our preliminary study of mass transfer measurement behind the biased orifice $\varepsilon=0.7 \%$ combined with a swirling flow at $S=0.23$ [14].

The increase in mass transfer behind the orifice is due to the growth of flow turbulence, which has been observed in the PIV measurement of the flow field behind the orifice [13]. On the other hand, the presence of the swirling flow promotes the flow turbulence behind the orifice and results in the growth of mass transfer in the pipe flow [13]. On the contrary, the velocity field behind the orifice is modified by the orifice bias for generating the biased flow directed to the shorter orifice side, which results in an increase in the mass transfer on the shorter orifice side. It should be mentioned that the generation of the biased flow behind the orifice is greatly contributed by the instability of the swirling flow through the orifice [13].

Figure 8(a) shows the variations of the maximum Sherwood number distribution on the shorter orifice side with respect to the swirl intensity $S$ and the orifice bias $\varepsilon$. The results are obtained from the present measurements for various combinations of swirl intensities and orifice biases. Note that $\varepsilon$ is the orifice bias normalized by the pipe diameter. The result on the shorter orifice side indicates that the maximum Sherwood number increases, when both the swirl intensity and the orifice bias become larger. The largest Sherwood number is found in the region of swirl intensity $S>0.2$ and the orifice bias $\varepsilon>0.4 \%$. It should be mentioned that the maximum Sherwood number is increased more than $40 \%$ by the combined effect of swirl intensity and orifice bias on the shorter orifice side. On the other hand, the maximum Sherwood number distribution on the longer orifice side shows the moderate magnitude of 

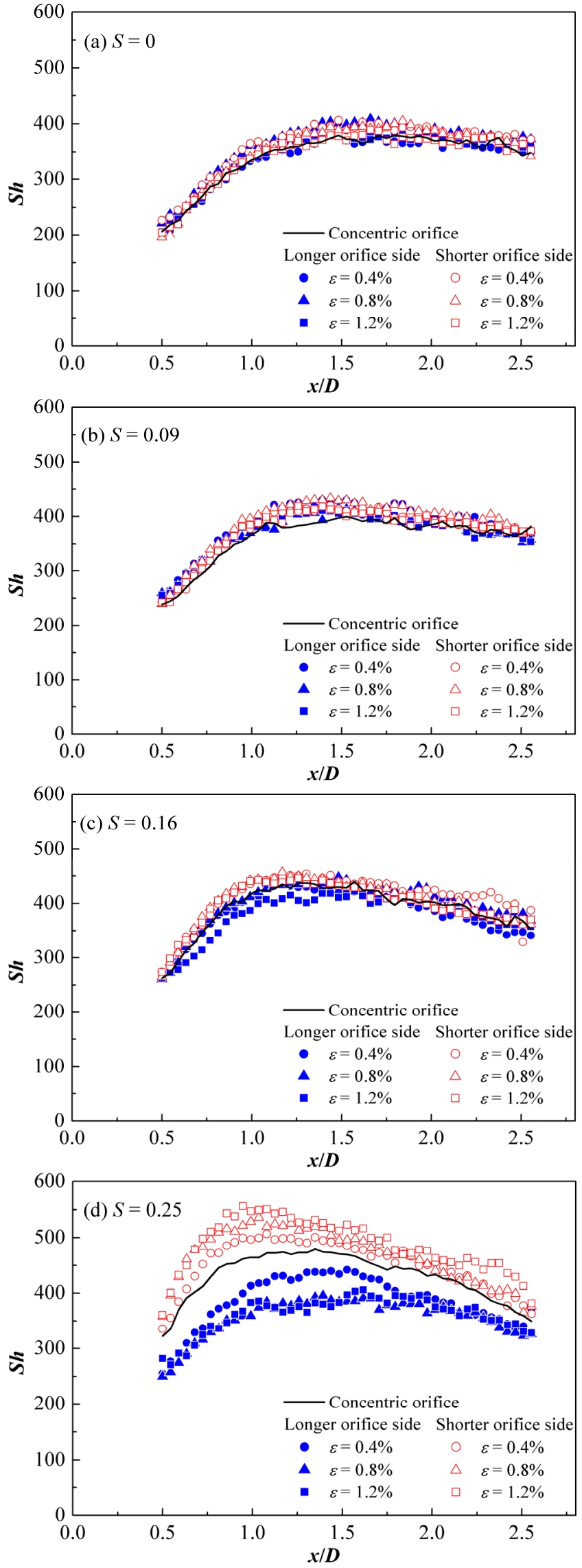

Fig. 7 Sherwood number distributions behind biased orifices in swirling flows $(\operatorname{Re}=1.9 \times$ $10^{4}$ ): (a) $S=0$; (b) $S=0.09$; (c) $S=0.16$; (d) $S=0.25$ 


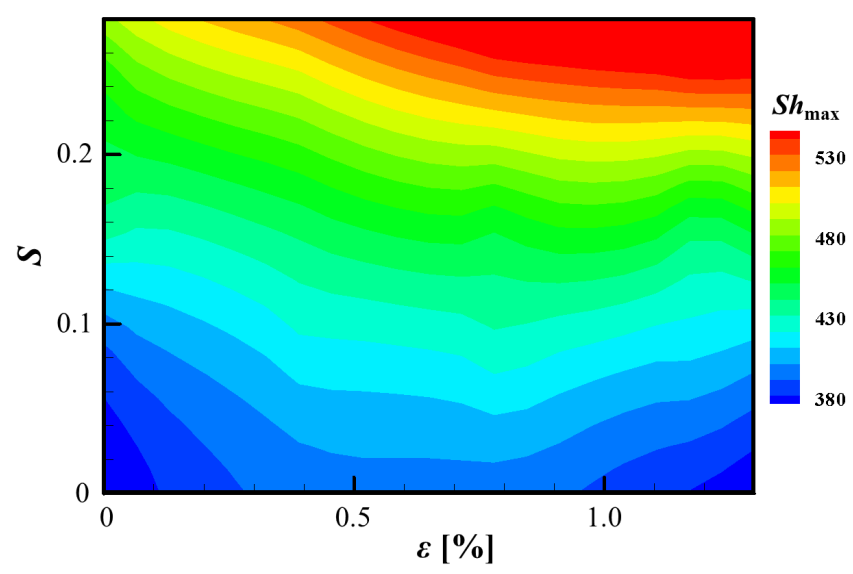

(a) Shorter orifice side

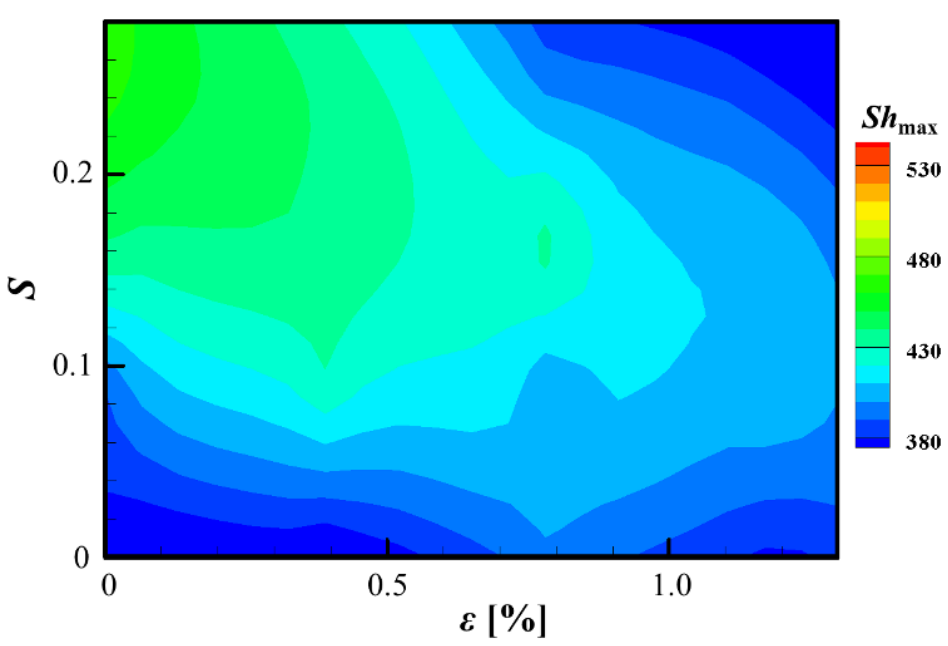

(b) Longer orifice side

Fig. 8 Maximum Sherwood numbers with respect to swirl intensity $S$ and orifice bias $\varepsilon$

mass transfer behind the orifice without orifice bias, and the small magnitude of mass transfer is observed in the region $S>0.2$ and $\varepsilon>0.8 \%$ (see Fig. 8(b)). Therefore, the degree of asymmetry in mass transfer can be found in this region. This consideration suggests that the critical condition for the occurrence of asymmetric mass transfer in the flow behind the orifice can be found in the region $S>0.2$ and $\varepsilon>0.8 \%$. This result indicates that the diameter error of the standard pipeline (JIS3456) is critical for the occurrence of asymmetric pipe-wall thinning, so that the swirl intensity has to be controlled smaller than 0.1 to avoid the asymmetric pipe-wall thinning in the pipe behind the orifice.

\section{Conclusions}

The combined effects of the swirling flow with the orifice bias on the pipe-wall thinning behind an orifice in a circular pipe were investigated to understand the condition for the occurrence of asymmetric pipe-wall thinning. In order to obtain the condition, the mass transfer measurements were carried out behind the orifice in a circular pipe by naphthalene sublimation method in wind tunnel for various combinations of swirl intensity and orifice bias. The asymmetric mass transfer was found behind the orifice with a bias larger than $0.4 \%$ of the pipe diameter, when the swirl intensity became larger than approximately 0.2 . The maximum Sherwood number was increased more than $40 \%$ in the present experiment by the combined effect of the swirl intensity and orifice bias. The role of the swirling flow was to enhance the mass transfer behind the orifice, while that of the orifice bias was to 
trigger the generation of the asymmetric flow behind the orifice. The mass transfer increased on the shorter orifice side and was decreased on the longer orifice side in the asymmetric mass transfer condition. The asymmetric mass transfer in the pipe behind the orifice can be suppressed by controlling the swirl intensity smaller than 0.1 .

\section{Acknowledgement}

The authors would like to express thanks to the members of Tohoku Cluster for the discussion on the experimental results. This work was supported by the project for the safety and maintenance of aged nuclear power plants by Safety Board of Japan in the fiscal year of 2006-2010.

\section{References}

[1] NISA, Secondary piping rupture accident at Mihama power station, unit 3, of the Kansai Electric Power Co. Inc. (2005), http://www.meti.go.jp/committee/materials/ g40906aj.html (in Japanese).

[2] H. Keller, Erosionskorrosion an nassdampfturbinen, VGB-Kraftwerkstechnik, Vol. 54 (1974), p. 292-295.

[3] T. Sydberger and U. Lotz, Relation between mass transfer and corrosion in a turbulent pipe flow, Journal of Electrochemical Society, Vol. 129, No. 2 (1982), pp. 276-283.

[4] L.E. Sanchez-Caldera, The mechanism of corrosion-erosion in steam extraction lines of power station, Ph.D. Thesis, Department of Mechanical Engineering, Massachusetts Institute of Technology, 1984, Cambridge, Massachusetts.

[5] JSME, Codes for power generation facilities, Rules on pipe wall thinning management, JSME S CA1-2005 (2005), 13-47 (in Japanese).

[6] S. Uchida, Latest experience with water chemistry in nuclear power plants in Japan. Power Plant Chemistry, Vol. 8 (2006), pp. 282-292.

[7] K. Yoneda and R. Morita, Investigation of flow characteristics affecting on pipe wall thinning, Part 1 Turbulent properties at orifice downstream in single phase flow, CRIEPI Research Report, No. L05007 (2006), pp. 1-23 (in Japanese).

[8] A. Nakamura, M. Murase, Y. Utanohara and Y. Nagaya, The effect of local flow field on flow accelerated corrosion, The background of the research and measurements of corrosion rate after an orifice, INSS Journal, Vol. 15 (2008), pp. $78-87$ (in Japanese).

[9] K. Yoneda, R. Morita, M. Satake and K. Fujiwara, Quantitative evaluation of effective factors on flow accelerated corrosion, Part 3 Proposition of wall thinning prediction model, CRIEPI Research Report, No. L08016 (2008), pp. 1-33 (in Japanese).

[10] J. Xiong, S. Koshizuka and M. Sakai, Turbulence modelling for mass transfer enhancement by separation and reattachment with two-equation eddy-viscosity models. Nuclear Engineering and Design, Vol. 241(2011), pp. 3190-3200.

[11] N. Fujisawa and H. Yamamoto, Occurrence of asymmetric flow pattern behind a double orifice in a square pipe, Journal of Visualization, Vol. 12 (2009), pp. 93-94.

[12] M. Ohkubo, S. Kanno, T. Yamagata, T. Takano and N. Fujisawa, Occurrence of asymmetrical flow pattern behind an orifice in a circular pipe, Journal of Visualization, Vol. 14 (2011), pp. 15-17.

[13] M. Ohkubo, T. Yamagata, S. Kanno and N. Fujisawa, Investigation on pipe-wall thinning by flow accelerated corrosion, Occurrence of asymmetrical flow by combined effect of swirling flow and orifice bias, Transactions of the JSME, Series B, Vol. 77 (2011), pp. 386-394 (in Japanese). 
[14] T. Takano, T. Yamagata, Y. Ito and N. Fujisawa, Investigation on pipe-wall thinning by flow-accelerated corrosion, Mass transfer phenomenon by combined effect of swirling flow and orifice bias, Maintenology, Vol. 10 (2011), pp. 30-35 (in Japanese).

[15] R.J. Goldstein and H.H. Cho, A review of mass transfer measurements using naphthalene sublimation, Experimental Thermal and Fluid Science, Vol. 10 (1995), pp. 416-434.

[16] D. Ambrose, I.J. Lawrenson and C.H.S. Sparke, The vapor pressure of naphthalene, Journal of Chemical Thermodynamics, Vol. 7 (1975), pp. 1173-1176.

[17] K. Aoki, Y. Nakayama and M. Wakatsuki, Study on the cylindrical combustor flow with swirling flow, 1st Report Characteristics of flow pattern for swirl number, Transactions of the JSME, Series B, Vol. 51 (1985), pp. $2759-2766$ (in Japanese).

[18] B.S. Petukhov, Heat transfer and friction in turbulent pipe flow with variable physical properties, Advances in Heat Transfer, Vol. 6 (1970), pp. 503-564. 